Michael Heine, Hansjörg Herr

\title{
Die beschäftigungspolitischen Konsequenzen von „Rot-Grün“
}

\section{Die Konturen eines neoliberalen rot-grünen Programms}

Mittlerweile zeichnen sich die Konturen rot-grüner Finanz- und Arbeitsmarktpolitik deutlicher ab als noch zu Zeiten eines Finanzministers Lafontaine. Als Einstieg in den Ausstieg einer alternativen Wirtschaftspolitik kann das gemeinsam von Schröder und Blair vorgelegte Papier Der Weg nach vorne für Europas Sozialdemokraten angesehen werden. Dort werden Massnahmen zur Haushaltskonsolidierung, Senkung der Unternehmenssteuern und Flexibilisierung der Arbeitsmärkte vorgeschlagen. Der finanzpolitische Teil wurde mittlerweile durch das Zukunftsprogramm 2000 umgesetzt. Dieses Programm sieht eine zusätzliche Kürzung der Ausgaben im Bundeshaushalt um $30 \mathrm{Mrd}$. DM gegenüber der von Waigel vorgelegten Finanzplanung vor. Doch damit nicht genug: „Die rot-grüne Sparpolitik soll konsequent fortgesetzt werden. Bundeskanzler Schröder und Finanzminister Eichel machten ... deutlich, dass sie von ihrem Kurs nicht abgehen werden. Eichel sagte, sein Sparpaket für den Haushalt 2000 sei erst der Anfang. 'Wir müssen bei der Neuverschuldung auf Null kommen.' Schröder sicherte Eichel seine Unterstützung zu. ... Unabdingbar sei die konsequente Durchsetzung des Sparprogramms. 'Es darf jetzt nicht gewackelt werden." (Tagesspiegel vom 26.7.1999, S.1). Und Wirtschaftsminister Müller machte die Ziele der Sparpolitik deutlich: „Man kann damit wirtschaftliche Grundsätze, wie das Zurückdrängen des Staates, gut verbinden“ (Tagesspiegel vom 25.7.1999, S.4). Auf diesem Politikfeld strebt die neue Regierung offensichtlich Kontinuität an, wenngleich sie mit dem Zukunftsprogramm 2000 das Tempo der Konsolidierungsbemühungen gegenüber der Kohl-Regierung deutlich zu erhöhen beabsichtigt.

Ähnlich stellt sich die Beschäftigungspolitik dar. Hatte man in der Koalitionsvereinbarung noch eine aktive Arbeitsmarkt- und Beschäftigungspolitik verabredet, so wird gegenwärtig eine konjunkturstabilisierende Fiskalpolitik zur Stabilisierung des Arbeitsmarktes - wie die Sparbemühungen zeigen - strikt abgelehnt. Ein Lieblingskind zumindest des grünen Koalitions- 
partners ist die Ökosteuer, die gleichzeitig die Beschäftigung erhöhen und den Energieverbrauch senken soll: Die Einnahmen aus der Ökosteuer sollen zur Absenkung der Lohnnebenkosten benutzt werden, und da Arbeit dann relativ billiger geworden ist, wird eine Erhöhung der Beschäftigung erwartet. Damit zeigt sich, dass auch die neue Bundesregierung von einem inversen Zusammenhang zwischen Lohnhöhe und Beschäftigung ausgeht, so dass sinkende Löhne ceteris paribus mit steigender Beschäftigung einhergehen sollen. Dem entspricht, dass Vorschläge wie jener vom rheinlandpfälzischen Ministerpräsidenten Kurt Beck (SPD), zwei Lohnnullrunden zwischen den Tarifparteien zu vereinbaren, um die Produktivitätsfortschritte für zusätzliche Arbeitsplätze zu nutzen, von führenden Vertretern der Koalition eher wohlwollend zur Kenntnis genommen werden. ${ }^{1}$

Damit haben sich führende Vertreter der Regierungsparteien die Krisendiagnose vor allem der Wirtschaftsverbände, aber auch der großen Mehrzahl der Wirtschaftsjournalisten zu eigen gemacht, wonach die Massenarbeitslosigkeit letztlich aus einem hierzulande zu hohen Lohnniveau resultiert. Die insbesondere von gewerkschaftlicher Seite vorgetragenen Hinweise, dass Löhne nicht nur Kosten, sondern auch eine wesentliche Nachfragekomponente darstellen, wurden im Verlauf der letzten Jahre zunehmend in den Hintergrund gedrängt. ${ }^{2}$

Allerdings wird - ebenso wie unter der Kohl-Regierung - die Tarifautonomie auch durch die gegenwärtige Regierungskoalition gewahrt. Zugleich zeigen die faktisch akzeptierten Aufweichungen des Ladenschlussgesetzes, dass auch die gegenwärtige Bundesregierung zu einer grösseren Flexibilität auf dem Arbeitsmarkt kommen möchte, ohne freilich die in der Tat unübersehbaren Missbräuche, wie sie im Rahmen der 630-Mark-Beschäftigung oder der Scheinselbständigkeit an der Tagesordnung waren, so zu akzeptieren.

Freilich lässt sich aus den obigen Ausführungen nicht ableiten, dass es keinerlei Unterschied zur Vorgängerregierung gibt. Reformvorhaben wie die ökologische Steuerreform, die steuerliche Entlastung kleiner und mittlerer Einkommen, die ab Mitte 2000 wirksam werden, die Erhöhung des Kindergeldes oder die Einführung des 630-Mark-Gesetzes und die gesetzliche Festlegung von Scheinselbständigkeit setzten andere Akzente als die alte Koalition. Dessen ungeachtet konzentriert sich Rot-Grün spätestens seit

1 Das Argument der lohnpolitischen Nullrunde schliesst vordergründig an die vorgeschlagenen Nullrunden bei den Altersrenten an. Die zweijährige Nichterhöhung der realen Rentenbezüge hat das Ziel, die Renten im Vergleich zu den Löhnen abzusenken und so die Rentenzahlungen der aktiv Beschäftigten zu senken. Herr Beck hat wohl nicht bemerkt, dass er mit seinem Vorschlag das Konzept der (relativen) Rentenabsenkung, das Minister Riester geschickt eingefädelt hatte, zerstört.

2 Allerdings ist auch der Konsumnachfrageansatz unzureichend, um Fehlentwicklungen auf dem Arbeitsmarkt adäquat zu. 
Mitte dieses Jahres auf wirtschaftspolitische Konzepte, die schon die Regierung Kohl über Jahre versucht hat umzusetzen. Von einem noch im Wahlkampf angekündigten Bruch zugunsten einer diesen Namen verdienenden ,alternativen“ Wirtschaftspolitik ist in der Tat nichts zu spüren. Einen generellen Unterschied gibt es jedoch: Rot-Grün scheint radikaler und wesentlich schneller eine Reihe neoliberaler Konzepte umzusetzen, die die Kohl-Regierung zwar in ihren Sonntagsreden anstrebte, politisch jedoch nur ansatzweise durchzusetzen vermochte.

Indem die neue Regierung im Kern beschäftigungspolitisch das gleiche tut wie die alte, und hierfür von zahlreichen Wirtschaftswissenschaftlern auch noch gelobt wird, stellt sich die Frage, ob Sparpolitik und Lohnzurückhaltung gleichsam die ehernen Gesetze erfolgreicher Beschäftigungspolitik sind. Wir konzentrieren uns im Folgenden auf die Beschäftigungspolitik und sparen die Konsequenzen und Erfolgsbedingungen einer Konsolidierungspolitik aus.

Um zu einer grösseren Flexibilität auf dem Arbeitsmarkt zu kommen, werden zahlreiche, in ihren Auswirkungen recht unterschiedliche Massnahmen gefordert. Überhaupt werden unter Flexibilisierung ganz verschiedene Sachverhalte verstanden, die in ihren ökonomischen Auswirkungen auch unterschiedliche Effekte haben. Diese verschiedenen Dimensionen der Flexibilisierung des Arbeitsmarktes sollen im folgenden diskutiert werden. Teilweise sind die geforderten bzw. schon eingetretenen Flexibilisierungen dysfunktional und für Stabilität der Ökonomie gefährlich, teilweise sind sie ungerecht in dem Sinne, dass sie die Ungleichheit in der Gesellschaft erhöhen. Alle folgend angesprochenen Dimensionen sind in der derzeitigen wirtschaftspolitischen Diskussion zu finden und werden teils aktiv gefordert, teils setzen sie sich marktmässig durch, ohne dass die Politik dagegen etwas unternimmt.

\section{Die Senkung des allgemeinen Lohnniveaus}

Im Zentrum der Diskussion um „neue“" ${ }^{3}$ beschäftigungspolitische Wege steht die Senkung des allgemeinen Lohnniveaus. Besonders attraktiv erscheint vielen Diskutanten die Senkung der Lohnnebenkosten, um so deutliche Abstriche beim individuellen Haushaltseinkommen zu vermeiden und zugleich den Produktionsfaktor Arbeit zu verbilligen. Dem entspricht, dass die Mehreinnahmen aus der Ökosteuer zur Reduzierung der Rentenbeiträge eingesetzt werden sollen. In die Logik einer Absenkung des Lohnniveaus zugunsten von mehr Beschäftigung passen auch Vorschläge a la Beck, wonach eine Lohnerhöhung unterhalb der Produktivitätsentwicklung empfoh-

3 Dass Vorstellungen dieser Art als innovativ und neuartig bewertet werden, ist im hohen Masse verwunderlich, da faktisch keines der folgenden Argumente jünger als 100 Jahre ist. 
len wird, um über sinkende Lohnstückkosten zu einer Lohnkostenentlastung bei den Unternehmen zu kommen. ${ }^{4}$ In jedem Fall wird davon ausgegangen, dass eine Verbilligung des Produktionsfaktors Arbeit zu seinem Mehreinsatz in der Produktion führt.

Selbstverständlich würden auch Vertreter neoklassischer Arbeitsmarkttheorie nicht bestreiten, dass neben der Lohnhöhe auch andere Faktoren das Beschäftigungsniveau beeinflussen, wie beispielsweise Wechselkursveränderungen, konjunkturelle Entwicklungen auf ausländischen Märkten oder staatliche Interventionspolitik. Gleichwohl wird die entscheidende Determinante aber in der Lohnhöhe gesehen.

So plausibel diese Annahme auf den ersten Blick auch sein mag, hält sie doch weder theoretischen noch empirischen Überprüfungen stand. Im Rahmen der sogenannten Cambridge-Cambridge-Debatte konnten Kritiker der neoklassischen Makroökonomie zweifelsfrei zeigen, dass der bis dahin behauptete eindeutige Zusammenhang zwischen Reallohnhöhe, Technikwahl $^{6}$ und Beschäftigung nur in einer Modellwelt mit einem einzigen Gut aufrecht zu erhalten ist, auch dann, wenn man alle übrigen Modellannahmen der neoklassischen Makroökonomie vorbehaltlos akzeptiert. ${ }^{7}$ Selbst wenn man nur zwei Kapitalgüter und damit unterschiedliche Kapitalintensitäten zwischen zwei Branchen annimmt, gibt es keinen theoretisch eindeutig ableitbaren Zusammenhang mehr zwischen Lohnund Beschäftigungshöhe. Es kann daher nicht mehr behauptet werden, dass sinkende Reallöhne zwingend zu einer Substitution von Kapital zugunsten von Arbeit und folglich zu steigender Beschäftigung führen würden. Ein hohes Beschäftigungsniveau ist sowohl mit niedrigen als auch mit hohen Reallöhnen möglich und ein Sinken der Reallöhne muss keinesfalls zu einer arbeitsintensiveren Technik und damit zu mehr Beschäftigung führen. Dies hat kein geringerer als der Nobelpreisträger für Ökonomie, Paul

4 Bei der Berechnung der Lohnstückkosten werden die nominalen Stundenlöhne ins Verhältnis zum realen Output je Arbeitsstunde (Stundenproduktivität) bzw. je Erwerbstätigen gesetzt. Insofern spiegeln sie - bei allen methodischen Schwierigkeiten der Berechnung die Lohnkosten je Einheit Output wider. Steigen die Löhne um den gleichen Prozentsatz wie die Arbeitsproduktivität, bleiben die Arbeitskosten konstant. Steigen sie langsamer, sinken die Arbeitskosten. Die Lohnstückkosten werden bei internationalen Vergleichen oftmals als Indiz für Wettbewerbsfähigkeit genutzt. Jedoch muss hier berücksichtigt werden, dass bei einem solchen Vergleich der Wechselkurs zu berücksichtigen ist, der sich viel schneller und stärker ändern kann als die nominellen Stundenlöhne.

5 Dieser Vorstellung liegt die neoklassische Arbeitsmarkttheorie zugrunde, wonach die gewinnmaximale Beschäftigungsmenge dann erreicht wird, wenn der Reallohnsatz dem physischen Ertrag des Grenzarbeiters entspricht. Da nun unterstellt wird, dass mit zusätzlicher Beschäftigung der physische Ertrag des Grenzarbeiters sinkt, kann nur bei sinkendem Reallohnsatz die Beschäftigung erhöht werden.

6 Es ist hier die Wahl der Unternehmen aus dem „Buch“ existierender Techniken gemeint. Selbstverständlich kommen bei Erfindungen zusätzliche Techniken zu den alten hinzu.

7 Dies kann im Rahmen dieses knappen Beitrags selbstverständlich nicht weiter begründet werden. Vgl. ausführlich hierzu Heine/Herr (1999, Kap. 3.2 und 3.3). 
geringerer als der Nobelpreisträger für Ökonomie, Paul Samuelson, frank und frei eingestanden, obwohl gerade er sich seinerzeit sehr für die Verteidigung der neoklassischen Makroökonomie ins Zeug gelegt hatte (vgl. Samuelson 1966). ${ }^{8}$ Im Ergebnis kann die Neoklassik kein makroökonomisches Modell ihrer „Realsphäre“ vorlegen, das einen eindeutigen Zusammenhang zwischen Lohnhöhe, Technikwahl und Beschäftigungsniveau begründen könnte, so daß sich daraus dann auch keine Politikempfehlung ableiten läßt.

Ein methodisch haltbares und widerspruchfreies Modell der „Realsphäre“ kann die neoklassische Ökonomie lediglich auf der Ebene der Mikroökonomie vorweisen. Werden die mikroökonomischen Ergebnisse dann in einem sogenannten ,walrasianischen Totalmodell“" zusammengefaßt, bleiben jedoch auch hier die gesamtwirtschaftlichen Zusammenhänge zwischen Lohnsenkung und Beschäftigungszunahme offen. Zwar kann im Kontext einer partiellen Arbeitsmarktanalyse begründet werden, dass ein Überschussangebot - hier also Arbeitslosigkeit - Preissenkungen verlangt, allerdings bleiben die Konsequenzen für die Ergebnisse der Totalanalyse unbestimmt. So führt eine Senkung der Reallöhne auf dem Arbeitsmarkt zu Preis-Mengen-Veränderungen auf allen anderen Märkten, was Einfluß auf die Produktionsentscheidungen hat, die sich wiederum auf den Arbeitsmarkt auswirken. Im Ergebnis bleibt offen, welche relativen Preise und welche Preis-Mengen-Verhältnisse sich letztlich in der Volkswirtschaft ergeben. Insofern ist die neoklassische Mikroökonomie zwar logisch widerspruchsfrei, kann aber keine beschäftigungspolitischen Aussagen machen. Eine Reihe führender neoklassischer Ökonomen kritisiert daher auch den immer wieder behaupteten schlichten Zusammenhang zwischen Reallohnund Beschäftigungsniveau (vgl. Bliss 1975, Hahn 1982 oder 1984). Es zeigt sich - und dies kommt in den beschäftigungspolitischen Debatten leider immer wieder zu kurz - dass die Begründungen für eine Lohnsenkungen zur Erhöhung des Beschäftigungsniveaus in einer Volkswirtschaft innerhalb des neoklassischen Paradigmas nur durch äußerst restriktive Annahmen abzuleiten sind - wie etwa die, daß die gesamte Ökonomie nur ein Gut kennt. ${ }^{10}$ Dass derartige Forderungen gleichwohl als praxisrelevantes

8 Die Argumentation betrifft auch die Wirksamkeit der ökologischen Steuerreform bezüglich ökologischer Effekte. Verändert eine Ökosteuer die relativen Preise für Arbeit und Energie, dann wird sich in der gesamten Ökonomie die Struktur der relativen Preise ändern. Dies führt zu einer neuen Technikwahl bei den Unternehmen. Ob die neuen Techniken dann insgesamt umweltfreundlicher sind oder nicht kann nicht allgemein begründet werden.

9 Auf monetärer Ebene liefert die Neoklassik mit der Quantitätstheorie des Geldes zwar eine umstrittene, jedoch methodisch saubere makroökonomische Theorie.

10 Das Problem besteht nicht darin, daß überhaupt mit restriktiven Annahmen gearbeitet wird, dies geschieht in jedem Modell, sondern, daß es überhaupt keinen Weg gibt, um 
Resultat wissenschaftlicher Anstrengungen durchgehen, entbehrt wirtschaftswissenschaftlich jeder Grundlage.

Auf eine weitere methodische Schwachstelle des neoklassischen Modells hat Keynes bereits vor mehr als 50 Jahren hingewiesen. Danach können Arbeitnehmer und Gewerkschaften selbst dann nicht zu einer Senkung der Reallöhne beitragen, wenn sie dies z.B. aus beschäftigungspolitischer Verantwortung gerne täten. Denn sie haben auf die Reallöhne keinen Einfluss, da sie stets nur über Nominallöhne verhandeln können. Die Reallöhne ergeben sich erst im nachhinein, wenn die Veränderungen des Preisniveaus feststehen. ${ }^{12}$ Sie sind das Ergebnis von Marktprozessen und nicht von Verhandlungsrunden. Eine Senkung der Tariflöhne bzw. eine Anhebung unterhalb der Produktivitätsfortschritte führen bei den Unternehmen zu Kostenentlastungen. Warum sollten die unter Wettbewerbsbedingungen agierenden Unternehmen nicht gezwungen werden, diese Kostensenkungen in Form von Preissenkungen weiterzugeben, so dass sinkende Nominallöhne nicht zu steigender Beschäftigung, sondern zu einem sinkenden Preisniveau führen? Was bei indirekten Steuern wie der Mehrwert-, Mineralöloder Tabaksteuer von jedem Betrachter ohne Wimpernzucken anerkannt wird, dass nämlich ihre Erhöhung (bzw. Senkung) an die Käufer weitergegeben wird, soll für einen anderen Kostenfaktor nun keine Gültigkeit mehr haben? Folgerichtig wird im Rahmen eines keynesianischen Gleichgewichtsmodells das Preisniveau über die Arbeits-, Kapital- und Bodenkosten bestimmt. ${ }^{13}$ Der skizzierte Zusammenhang zwischen Lohnhöhe und Preisniveau gilt selbstverständlich auch für die Lohnnebenkosten, so dass durch die Verwendung der Mitteleinnahme aus der ökologischen Steuerreform zugunsten einer Senkung der Rentenbeiträge keine Beschäftigungseffekte zu erwarten sind, sondern eine Senkung des Preisniveaus.

Vor diesem Hintergrund zeigt sich, dass eine Senkung des Lohnniveaus nicht zu mehr Beschäftigung führt, sondern die Gefahr einer Deflation heraufbeschwört. ${ }^{14}$ Deflationen wirken zerstörerisch auf Ökonomien, da ein sinkendes Preisniveau die Realverschuldung der Unternehmen erhöht und

sich von diesem Modell aus, der Realität auch nur minimal anzunähern: geht man von der Ökonomie mit einem Kapitalgut auch nur zu einer Ökonomie mit zwei Kapitalgütern über, ist von den Ergebnissen des ersten Modells nichts mehr zu retten.

11 Warum wissenschaftlich obsolete Ideen wirtschaftspolitisch gleichwohl äusserst relevant geblieben sind, kann im Rahmen dieses Aufsatzes nicht diskutiert werden. Vgl. dazu Gordon (1995), der eine ganze Reihe von Faktoren anführt, die hier eine Rolle spielen.

12 Der Reallohnsatz ist definiert als Nominallohnsatz dividiert durch den Preisindex, so dass ein steigendes (sinkendes) Preisniveau den Reallohnsatz senkt (anhebt).

13 Im Ungleichgewicht führen ceteris paribus Angebotsüberschüsse zu Preisniveausenkungen und Nachfrageüberschüsse zu Preisniveauerhöhungen.

14 Lohnabschlüsse oberhalb der Produktivitätsentwicklung führen demnach auch nicht zu einer Umverteilung auf Kosten der Unternehmen, sondern über eine Lohn-Preis-Spirale zur Inflation. 
damit Tilgungen und Zinszahlungen erschwert. Werden einzelne Unternehmen aus diesem Grunde zahlungsunfähig, können Kreditketten mit entsprechend krisenhaften Folgen reissen. Die Weltwirtschaftskrise Ende der zwanziger Jahre liefert dazu das Anschauungsmaterial.

Wenn es keinen Zusammenhang zwischen Lohn- und Beschäftigungsniveau gibt, stellt sich die Frage, von was die Nachfrage nach Arbeit dann abhängt. Sie resultiert nach keynesianischer Auffassung im Kern aus Entscheidungen, die auf den Vermögensmärkten gefällt werden. Denn hier wird das Investitionsniveau festgelegt. Bei gegebenem Zinsniveau entscheiden die Gewinnerwartungen der Unternehmen über die Nachfrage nach Investitionsgütern und damit über das Produktionsniveau und die Einkommensbildung. In die Gewinnerwartungen gehen unterschiedliche Parameter ein wie Erwartungen über den künftigen Absatz, die Preis- und Zinsentwicklungen oder wirtschaftspolitische Weichenstellungen. In einem theoretischen Modell müssen Erwartungen als exogene Grösse gesetzt werden, da sie objektiv nicht schlüssig ableitbar sind. Dies schliesst nicht aus, dass in einer historisch-spezifischen Situation über Erwartungen Aussagen möglich sind. Die Investitionsentscheidungen bestimmen - wie gesagt - das Niveau der Produktion und der Einkommensbildung und damit auch die Konsumgüternachfrage. Ist der Umfang der Produktion festgelegt, fragen die Unternehmen bei gegebener Technik die entsprechend benötigten Arbeitskräfte nach. Ob arbeitsintensivere oder kapitalintensivere Techniken gewählt werden, kann nicht allgemein gesagt werden. Auf alle Fälle gibt es in einem allgemeinen theoretischen Modell keine Beziehung zwischen sinkender Lohnhöhe bzw. steigenden Zinssätzen und der Wahl einer arbeitsintensiveren und damit einer die Beschäftigung stärker fördernden Technik (vgl. oben). Die Differenz zwischen dieser Arbeitsnachfrage und dem Arbeitsangebot bildet die unfreiwillige Arbeitslosigkeit. Die Nominallöhne werden als Kostenfaktor auf die Preise überwälzt und haben folglich auf die nachgefragte Beschäftigungsmenge keinen Einfluss (vgl. dazu ausführlich Heine/Herr 1999, Kap. 4.3-4.6).

Die theoretische Brüchigkeit des behaupteten Zusammenhangs von Lohnund Beschäftigungshöhe spiegelt sich auch in empirischen Studien wider, wenngleich man zugestehen muss, dass empirische Feststellungen keine strengen Beweise für die Angemessenheit bzw. Unangemessenheit eines theoretischen Modells liefern können, sondern allenfalls mehr oder weniger gute Hinweise. Unter Berücksichtigung dieser Einschränkung zeigen empirische Studien beispielsweise des DIW, dass es keinen Zusammenhang zwischen Lohnzurückhaltung und Beschäftigungszunahme gibt (vgl. DIW 1995 und 1997). ${ }^{15}$ Obwohl der Anstieg der Lohnstückkosten in West-

15 Sehr informativ sind die vom DGB zusammengestellten Arbeitsmarktdaten, DGB (1999). 
deutschland in den siebziger und achtziger Jahre geringer war als in den meisten anderen entwickelten Industrieländern, konnten die Beschäftigungsprobleme in der Bundesrepublik keinesfalls gelöst werden. Oder: In den USA sind die Reallöhne seit dem Beginn der achtziger Jahre nicht langsamer gestiegen als in Europa und trotzdem konnten dort vergleichsweise grosse Beschäftigungserfolge erzielt werden und zwar nicht nur im Bereich der „bad jobs“ (vgl. Flassbeck 1998). Schliesslich liegt die bereinigte Bruttolohnquote ${ }^{16}$ der Bundesrepublik mittlerweile wieder auf dem Niveau der frühen sechziger Jahre. Während wir heute jedoch mit dem Problem der Massenarbeitslosigkeit konfrontiert sind, herrschte seinerzeit Vollbeschäftigung.

Häufig wird auch eine Strategie lohnpolitischer Zurückhaltung gefordert, um die internationale Wettbewerbsfähigkeit zu erhöhen. Sinkende Lohnstückkosten sollen diesem Konzept zufolge die preisliche Wettbewerbsfähigkeit erhöhen. Freilich gilt dies nur bei unveränderten Wechselkursen, was meist unterschlagen wird. Eine Strategie der Erhöhung der internationalen Wettbewerbsfähigkeit über vergleichsweise moderate Erhöhungen der Lohnstückkosten wurde in der Bundesrepublik Deutschland seit den fünfziger Jahren verfolgt. Unter dem Regime fester Wechselkurse in den fünfziger und sechziger Jahren war diese Strategie äusserst erfolgreich. ${ }^{17}$ Allerdings wird sie seit den siebziger Jahren mit beschäftigungspolitisch zweifelhaftem Erfolg verfolgt. ${ }^{18}$ Denn unter den Bedingungen flexibler Wechselkurse führt eine solche Strategie zu Aufwertungstendenzen der eigenen Währung. Eine im internationalen Vergleich niedrige Inflationsrate und Leistungsbilanzüberschüsse regen nämlich üblicherweise Kapitalimporte an, die zu Aufwertungstendenzen führen. Im Ergebnis kann so das wettbewerbspolitische Ziel, das mit der Lohnzurückhaltung verfolgt wurde, nicht erreicht werden. Die Aufwertung kann den Effekt der Lohnzurückhaltung nicht nur aufheben, sondern sogar überkompensieren. So hat die Bundesrepublik Deutschland Anfang der sechziger, Ende der siebziger oder Anfang der neunziger Jahre Aufwertungskrisen durchlaufen, mit erheblichen negativen Konsequenzen für die Beschäftigung.

Dieser Aufwertungseffekt fällt unter den Bedingungen einer Einheitswährung wie dem Euro oder bei einem System absolut fester Wechselkurse

16 Die bereinigte Bruttolohnquote gibt den Anteil der Einkommen aus unselbständiger Arbeit am Bruttoinlandsprodukt an, wobei Strukturveränderungen zwischen den abhängig Beschäftigten und den Selbständigen berücksichtigt werden.

17 Allerdings kam es 1961 aufgrund äusserst hoher Leistungsbilanzüberschüsse der Bundesrepublik zu einer Aufwertung der D-Mark innerhalb des Systems von Bretton Woods.

18 In den neunziger Jahren lagen Nominallohn- und Preisniveauentwicklung in Deutschland eher im Mittelfeld aller entwickelten Volkswirtschaften, so dass in dieser Phase - unveränderte Wechselkurse unterstellt - die Lohnentwicklung die internationale preisliche Wettbewerbsfähigkeit nicht steigerte (vgl. International Monetary Fund 1998, S. 183). 
weg. Versuchen einzelne Länder unter dieser Bedingung die eigenen beschäftigungspolitischen Probleme über eine Politik der Lohnzurückhaltung auf die übrigen Mitglieder des Währungsraums abzuwälzen, so drohen Lohnsenkungswettläufe oder die währungspolitische Zusammenarbeit gerät in Gefahr, was schnell in einen Abwertungswettlauf münden kann. Beide Fälle sind katastrophal für Wachstum und Beschäftigung. Beispielsweise hat die Niederlande seit den achtziger Jahren nicht ohne Erfolg versucht, eine solche Politik im Rahmen des Europäischen Währungssystems (EWS) zu betreiben (vgl. Volz 1998). Dass die negativen Auswirkungen auf die Europäische Union eher gering blieben, liegt daran, dass die Niederlande ein relativ kleines Land sind und es innerhalb der Phase des EWS noch Wechselkursanpassungen gab. ${ }^{19}$ Würden in Deutschland Nullrunden bei den Löhnen stattfinden, käme es bei den anderen Teilnehmerländer der Europäischen Währungsunion zu einem enormen Anpassungsduck, der dann wiederum negativ auf Deutschland zurückschlagen würde.

Für starke Volkswirtschaften ist es allemal dubios, den Arbeitsmarkt mit aller Kraft über Leistungsbilanzüberschüsse entlasten zu wollen. Denn die Leistungsbilanzüberschüsse hier gehen dann einher mit Leistungsbilanzdefiziten in anderen entwickelten Volkswirtschaften und/oder den relativ unterentwickelten Ländern. Hohe Leistungsbilanzungleichgewichte zwischen den entwickelten Industrieländern tragen zu Währungsturbulenzen zwischen den dominierenden Weltwährungen bei und destabilisieren dadurch die Weltwirtschaft. Negative Leistungsbilanzen der unterentwickelten Länder führen zur Gefahr von Schuldenkrisen, die eine nachholende Entwicklung belasten oder gar verhindern. Es wäre für die Weltwirtschaft fatal und wenig erfolgversprechend, wenn die gesamte Europäische Union über Leistungsbilanzüberschüsse die Effekte eines internen Lohnsenkungswettbewerbs lösen wollte. Dass eine negative Leistungsbilanz einer starken Volkswirtschaft sehr wohl mit Beschäftigungserfolgen vereinbar ist, zeigen die USA. Für 1999 wird ein Rekorddefizit von über 300 Mrd. \$ erwartet, nach rund 230 Mrd. \$ 1998 und etwa 150 Mrd. \$ 1997. In keinem Jahr der neunziger Jahre konnten die USA eine positive Leistungsbilanz aufweisen (vgl. iwd 1999) und gleichwohl wurde Jahr für Jahr eine positive Beschäftigungsbilanz vorgelegt. Zugleich hat die amerikanische Ökonomie einen bedeutenden Beitrag zur Stabilisierung der Weltwirtschaft geleistet.

Als Ergebnis zeigt sich, dass eine Absenkung des allgemeinen Lohnniveaus zur Überwindung der Massenarbeitslosigkeit eine dysfunktionale Massnahme darstellt, da sie die Gefahr einer deflationären Entwicklung heraufbeschwört und die internationale Kooperation erheblich belastet. An einer

19 So haben England, Italien und eine Reihe kleinerer Länder in den Jahren 1992/93 erfolgreich abgewertet. 
produktivitätsorientierten Entwicklung des Lohnniveaus als wirtschaftspolitischem Desiderat führt demnach kein Weg vorbei.

\section{Die Aufhebung des Flächentarifvertrages}

Neben der produktivitätsorientierten Lohnpolitik wird von den Befürwortern einer flexiblen Arbeitsmarktpolitik auch der Flächentarif innerhalb einer Branche als Gift für mehr Beschäftigung bewertet. So drängen beispielsweise die Wirtschaftsverbände im Rahmen der Verhandlungen im „Bündnis für Arbeit“ darauf, die traditionelle Lohnfindung über Flächentarifvereinbarungen zu „modernisieren“. Im Ergebnis soll eine Lohnfindung zugunsten der Stärkung von betrieblichen Regelungen und auf Kosten von Flächentarifvereinbarungen für ganze Branchen vereinbart werden. Begründet wird dieser Vorschlag damit, dass wettbewerbsschwächere Unternehmen mit einheitlichen Tarifabschlüssen überfordert seien, so dass deren Existenz gefährdet werde oder zumindest Wachstumschancen verspielt würden. In beiden Fällen gingen Arbeitsplätze verloren.

Dieses Argument verwundert insofern, als es nicht in die neoklassische Marktlogik passen will. Exekutiert der Flächentarifvertrag doch gerade das Marktgesetz eines einheitlichen Preises. In Marktwirtschaften, so üblicherweise die neoliberale Position, ist es doch gerade die Aufgabe des Wettbewerbs, knappe Ressourcen an den „lahmen Enten“ vorbei und zu den effizientesten Unternehmen hin zu leiten. Nun plötzlich sollen die effizienten Unternehmen durch vergleichsweise hohe Tarifabschlüsse abgestraft und jene, die organisatorische und technologische Innovationen verschlafen haben, durch Lohnzurückhaltung gleichsam subventioniert werden. Jenseits dieser ordnungspolitischen Bedenken bleibt völlig unklar, wie es so zu den beschworenen kräftigen Beschäftigungseffekten kommen soll. Die Marktanteile, die die „Grenzanbieter“ verlieren, gewinnen die effizienteren Unternehmen hinzu. Sicherlich gehen begrenzt Arbeitsplätze verloren, da durch die Beseitigung der Grenzanbieter die Produktivität insgesamt erhöht wird. Die Vertreter der Auflösung des Flächentarifvertrages sollten jedoch dann offen sagen, dass sie durch Reduzierung der Innovationskraft und Produktivität das Beschäftigungsproblem mildern wollen. Wir betrachten eine solche Strategie als entwicklungshemmend und für eine Marktökonomie als dysfunktional. Insofern spricht nichts dagegen, allen Unternehmen einer Branche die gleichen tariflichen Rahmen- und damit Wettbewerbsbedingungen vorzugeben, damit der Markt Spreu von Weizen trennen kann. Aus keynesianischer Sicht ist die Auflösung der Flächentarife vor allem deshalb nicht akzeptabel, weil so die Unsicherheiten in einer Volkswirtschaft erhöht werden. Denn wenn Tarife auf betrieblicher Ebene ausgehandelt werden, kann das einzelne Unternehmen nicht wissen, was morgen 
beim Konkurrenten vereinbart wird. Sofern jener seine Mitarbeiter zu niedrigeren Abschlüssen bewegen kann, hat dieser Probleme. Folglich wird sich der Planungshorizont der Unternehmen verkürzen, weil sich die Unsicherheit erhöht. Eine Erhöhung der Unsicherheit führt aber ceteris paribus zu sinkender Investitionsbereitschaft und erweist sich damit als dysfunktional für Wachstum und Beschäftigung.

\section{Lohnspreizung innerhalb und zwischen Branchen}

Als ein weiteres „Patentrezept“ zur Entlastung des Arbeitsmarktes wird regelmässig eine stärkere Lohnspreizung sowohl innerhalb einer Branche als auch zwischen den Branchen angesehen. Innerhalb einer Branche liesse sich eine stärkere Lohndifferenzierung beispielsweise durch die Einführung von Leichtlohngruppen realisieren. Erwartet wird, dass die Unternehmen dann eine arbeitsintensive Technik wählen, die schlecht qualifizierten Arbeitnehmern eine Beschäftigungschance eröffnet. Eine niedrige Produktivität soll mit niedriger Entlohnung einhergehen, da ein schlecht bezahlter Arbeitsplatz besser sei als keiner.

Diesem Ziel dient auch die stärkere Einkommensdifferenzierung zwischen den Branchen. Die Beschäftigungshoffnungen dieser Flexibilisierungsvariante setzen darauf, dass Niedriglohnbranchen wie die "Schuhputz“- oder die „Dienstmädchenbranche“ prosperieren und Arbeitskräfte aufsaugen. Aufgegeben werden muss bei diesem Vorschlag die Idee eines relativ einheitlichen Lohnniveaus in allen Branchen.

Ein stärkeres Einkommensgefälle innerhalb einer Branche oder zwischen den Branchen lässt sich freilich nur etablieren, wenn die sozialen Sicherungssysteme abgebaut werden. Sofern Ersatzleistungen des Arbeitsamtes oder die Zuwendungen des Sozialamtes ähnlich hoch sind wie schlecht bezahlte Jobs, liegt die Vermutung nahe, dass sich Betroffene gegen die Arbeit und für die staatlichen Transfers entscheiden. Länder mit ausgeprägter Einkommensungleichheit zeichnen sich folgerichtig immer dadurch aus, dass staatliche Sozialleistungen entweder nie auf hohem Niveau ausgeprägt waren oder in den letzten Jahren drastisch abgebaut wurden.

Die Hoffnung, daß eine Lohnspreizung zu mehr Beschäftigung führt, beruht auf zwei Argumenten. Das erste Argument setzt darauf, dass die Lohnspreizung zu einer arbeitsintensiveren Technik führt. Da es zwischen Lohnniveau und Technikwahl keinen theoretisch begründbaren Zusammenhang gibt, gibt es auch zwischen Lohnspreizung und Technik keinen deterministischen Zusammenhang. Man unterstellt, dass die Unternehmen schon eine arbeitsintensive Technik finden und einsetzen werden, die bei entsprechend niedrigen Löhnen gewinnmaximierend ist. Freilich sind die Zusammenhänge zwischen Lohnniveau und Technikwahl - hierauf wurde 
bereits hingewiesen - komplexer als sie in diesem Modell unterstellt werden. Nehmen wir zur Illustration an, die Lackierer verzichten auf Lohn. Damit werden die Kosten und folglich die Preise von lackierten Waren billiger. Gehen diese Waren als Vorleistungen in andere Branchen ein, so verändern sich hier ebenfalls die Herstellungskosten und Preise. Im Ergebnis kommt die Volkswirtschaft zu einer völlig neuen Struktur relativer Preise. Welche Technik sich unter dieser neuen Struktur relativer Preise als gewinnmaximierend erweisen wird, ist unbekannt. Sie kann insgesamt arbeits-, aber eben auch kapitalintensiver sein. Man weiß einfach nicht, ob schlecht bezahlte Lackierer die hochtechnisierten Lackierereien der Automobilkonzerne tatsächlich verdrängen können und welches Lohnniveau dazu notwendig wäre. Unbekannt ist auch, welchen Einfluss Niedriglohngruppen auf den Herstellungspreis von hochtechnisierten Lackierereien haben. Niemand weiss es - dessen ungeachtet wird munter „,drauflos“ argumentiert und man läuft Gefahr, dass die im Prinzip bewährte Einkommenspolitik und die sozialen Sicherungssysteme zugunsten unsicherer Spekulationen geopfert werden.

Das zweite Argument beruht darauf, dass bestimmte Güter preisgünstiger angeboten werden können und aufgrund dieses Umstandes die Konsumnachfrage nach diesen Gütern steigt. Sinkt der Preis für Dienstmädchen, so das Argument, dann wird es mehr Privathaushalte geben, die Dienstmädchen nachfragen. Das Argument lässt sich auf den Lieferservice von Essen, Massagen zur Entspannung, Wachdienste oder das Parken von Autos vor Supermärkten ausweiten. Sicherlich sind bei einer deutlichen Zunahme der Lohnspreizung Beschäftigungseffekte in diesen Bereichen wahrscheinlich. Dies gilt insbesondere bei Dienstleistungen, die ohne Inputs von Kapitalgütern produziert werden können, also im wesentlichen persönliche Dienstleistungen. Gibt es keine Kapitalinputs, dann bestimmt der Preis der Arbeit allein den Preis der Dienstleistung. Gehen in die Dienstleistung jedoch Kapitalinputs ein, dann kann nicht mehr ausgeschlossen werden, dass trotz einer Lohnspreizung, die zunächst vermuten lässt, dass Lackierer Lackieranlagen verdrängen, die Lackieranlagen im Endeffekt so billig werden, dass die Lackierer das Nachsehen haben und nicht verstärkt nachgefragt werden. Über den Zusammenhang zwischen Ungleichheit der Entlohnung und Beschäftigungsentwicklung existieren in der Tat zahlreiche empirische Studien (vgl. Bosch 1998), die die vergangene Entwicklung festhalten. Eine umfangreiche Studie der OECD kommt zu dem bemerkenswerten Resultat:

„Es gibt nur wenig schlüssige Belege, die zeigen, dass Länder mit einem geringen Anteil an Niedrigbezahlten dies auf Kosten höherer Arbeitslosenzahlen oder einem geringeren Beschäftigungsniveau für besonders gefährdete Gruppen wie Jugendliche oder Frauen erreicht haben“ (OECD 1996: 76, zit. nach Bosch 1998: 18).

Insgesamt zeigt sich, dass es Länder mit sehr starker Einkommensdifferen- 
zierung gibt, die sich beschäftigungspolitisch sehr gut und andere, die sich sehr schlecht entwickelt haben. Das gleiche gilt für Länder mit eher geringen Einkommensunterschieden. Dem entsprechen empirische Befunde, wonach die Beschäftigungsentwicklung im wesentlichen von Makrodaten wie der verfolgten Geld- und Fiskalpolitik oder der Wechselkursentwicklungen abhängen und nicht vom Umfang der Lohnspreizung (vgl. Heise 1997). Dieser Befund passt wiederum gut zu den oben skizzierten theoretischen Argumenten.

Trotz dieser Befunde ist nicht auszuschliessen, dass durch den Abbau von sozialen Sicherungssystemen und der Etablierung eines Niedriglohnsektors einzelne zusätzliche Arbeitsplätze entstehen. Denn sehr niedrige Einkommen etwa im Dienstleistungssektor führen zu Billigangeboten vor allem im Bereich haushaltsorientierter Dienstleistungen (vom Schuhputzer bis zum Kindermädchen), so dass sich auch die Mittelschicht derartige Dienste leisten könnte. Freilich liegt die Vermutung nahe, dass die Beschäftigungseffekte eher gering bleiben dürften, da auf der Basis von 630-Mark-Jobs und Scheinselbständigkeiten ein solcher Markt längst auch in Deutschland existiert. Dessen ungeachtet bleibt die Frage, ob der Preis für einige „,bad jobs“ in Form zunehmender sozialer Ausdifferenzierungen, die von Armut trotz Erwerbstätigkeit über unzureichende Renten- und Krankenversicherung bis hin zur Wiedereinführung kirchlich organisierter ,Suppenküchen“ reichen können, nicht zu hoch ist.

Eng mit der Herausbildung eines Niedriglohnsektors sind die Tendenzen zur Auflösung des Normalarbeitsverhältnisses verbunden. Kennzeichnend für diesen Trend sind die sprunghafte Ausdehnung der 630-Mark-Jobs und der Scheinselbständigkeit. Unabhängig davon, ob der Arbeitsmarkt über diesen Weg nennenswert entlastet wird, führt diese Entwicklung dazu, dass die sozialen Sicherungssysteme erheblich unter Druck geraten. Werden beispielsweise keine Rentenbeiträge geleistet, so führt dies einerseits zu aktuellen Finanzierungsproblemen der Rentenversicherung, andererseits ist Altersarmut der Beschäftigten zu erwarten, die keine Rentenbeiträge zahlen. Dies führt dann zu Belastungen bei der Sozialhilfe.

\section{Regionale Lohnspreizung}

Unter einer regionalen Lohnspreizung werden unterschiedliche Lohnniveaus der Regionen innerhalb eines gemeinsamen Währungsraumes verstanden. Beispielsweise wird innerhalb des Eurolandes in Portugal durchschnittlich real weniger verdient als in Deutschland. Auch innerhalb Deutschlands unterscheiden sich die Durchschnittseinkommen etwa zwi- 
schen West- und Ostdeutschland. ${ }^{20}$ Dem liegt zugrunde, dass verschiedene Regionen eines Währungsraums unterschiedliche Produktivitäten aufweisen. Auf dieser Grundlage können die Löhne nicht flächendeckend einheitlich sein. Dies würde nämlich dazu führen, dass die Lohnstückkosten in Regionen mit Produktivitätsrückständen so hoch wären, dass diese Regionen nicht wettbewerbsfähig wären. Relativ zu hohe Einkommen würden mit Arbeitsplatzverlusten bezahlt werden. Diese Regionen würden zu Problemregionen, die auf Dauer kaum über Transfers zu stabilisieren wären. Insofern muss eine produktivitätsorientierte Lohnpolitik zwingend auf die regionalen Produktivitätsgefälle bzw. unterschiedliche Produktivitätsentwicklungen Rücksicht nehmen. Auch bei den neuen Bundesländern wäre eine langsamere Lohnangleichung an das westdeutsche Niveau wünschenswert gewesen, da dadurch der ökonomische Absturz in den neuen Ländern abgemildert und der Transferbedarf reduziert worden wäre. Gerade der deutsche Einigungsprozess zeigt, wie schwierig eine abgestimmte und damit funktionale regionale Lohnspreizung wirtschaftspolitisch umzusetzen ist. Schwierigkeiten sind auch innerhalb der Europäischen Währungsunion zu erwarten. Sie werden verschärft, wenn ökonomisch große und in der Produktivitätsentwicklung führende Länder wie Deutschland die Währungsunion mit Nullrunden bei der Lohnentwicklung belasten. Falls dann die anderen Länder nachziehen, wären Lohnsenkungswettläufe nicht mehr Sesłastscedrięßedlich wäre es wünschenswert, die Produktivitäts- und damit die Einkommensunterschiede perspektivisch einzuebnen. Denn dauerhafte Einkommensunterschiede zwischen Regionen führen unter anderem dazu, dass bei liberalisierten Arbeitsmärkten leistungsstarke Arbeitnehmer die Problemregionen verlassen. Indem diese Regionen so strukturell ,,ausbluten“, perpetuiert sich die Unterentwicklung. Eine ausreichende regionale Lohnspreizung zur Verteidigung der regionalen Wettbewerbsfähigkeit nachhinkender Regionen führt somit keinesfalls automatisch zu einer überregionalen Anpassung der Produktivitätsniveaus und Lebensstandards. Maßnahmen zur Förderung der Problemregionen - von einem Ausbau der Infrastruktur über Investitionsförderprogramme bis hin zu Finanzausgleichszahlungen - sind notwendig. Dessen ungeachtet weisen Agglomerationen üblicherweise sogenannte Agglomerationsvorteile auf, die regionalpolitisch kaum zu kompensieren sind. Daher lassen sich innerhalb aller Währungsräume Metropolisierungstendenzen einerseits und Peripherisierungstendenzen andererseits feststellen, die die Unterschiede zwischen Re-

20 Die regionalen Differenzen bei den Reallöhnen sind in aller Regel weniger stark ausgeprägt als die regionalen Differenzen bei den Nominallöhnen. Dies liegt daran, dass Güter und Dienstleistungen, die überregional nicht gehandelt werden (beispielsweise Wohnraum oder lokale Dienstleistungen), in nachhinkenden Regionen billiger sind. 
gionen nicht einebnen, sondern eher verstärken (vgl. Heine 1989). Theoretisch könnten regionale Produktivitätsunterschiede natürlich durch einen interregionalen Finanzausgleich kompensiert werden. Allerdings ist anzunehmen, dass die Subventionierung der weniger entwickelten Regionen und der damit einhergehende Transferbedarf den finanziellen Spielraum und die politische Akzeptanz der reicher Regionen auf Dauer übersteigt. Insofern wird man an regional gespreizten Löhnen nicht vorbeikommen. Anders als bei Lohnspreizungen zwischen Unternehmen einer Branche innerhalb der Regionen wird so das Unsicherheitspotential nicht erhöht. Insofern ist eine solche Lohnspreizung ökonomisch durchaus funktional.

\section{Fazit}

Als Fazit zeigt sich, dass wirtschaftspolitische Massnahmen, die die Massenarbeitslosigkeit über die Flexibilisierung des Arbeitsmarktes abzubauen gedenken, zum Scheitern verurteilt sind. Ein Teil der Flexibilisierungsmassnahmen - etwa die Senkung des Lohnniveaus - sind dysfunktional und ökonomisch gefährlich. Andere Flexibilisierungsmassnahmen - etwa die branchenmässige Lohnspreizung - sind in ihren Auswirkungen ungewiss, jedoch sozial ungerecht, da sie die Ungleichheit innerhalb der Gesellschaft direkt fördern.

Der Mangel der rot-grünen Vorstellungen zur Lösung des Beschäftigungsproblems liegt darin, dass die Lösung bei Reformen auf dem Arbeitsmarkt gesucht wird. Bei dieser Grundüberzeugung gibt es eine grosse Koalition, die von der derzeitigen rot-grünen Regierung über die ehemalige Regierung Kohl bis hin zu Teilen der Gewerkschaften reicht. Vergessen wird, dass diese Überzeugung Ausfluss nur eines ökonomischen Paradigmas ist, nämlich der Neoklassik. Schlimmer wiegt, dass den Vorstellungen ein eingeschränktes Modell zugrunde liegt, das sich Ende des letzten Jahrhunderts - wir schreiben diese Zeilen noch vor dem Jahr 2000 ! - herausgebildet hat, und das nach dem Zweiten Weltkrieg einen völligen theoretischen Schiffbruch erlitten hat.

Nach unserer Ansicht spiegelt der Arbeitsmarkt lediglich Entscheidungen wider, die auf den Vermögensmärkten gefällt wurden. Hier entscheidet sich das Niveau der Produktion, der Einkommensbildung, der aggregierten Gesamtnachfrage und schliesslich der Arbeitsnachfrage. Die Vorstellung, daß die Arbeitsnachfrage letzten Endes von der Lohnhöhe abhängt, verkennt die Logik einer geldgesteuerten Ökonomie. Folglich muss die Bekämpfung der Arbeitslosigkeit letztlich am Vermögensmarkt ansetzen. Daran geht kein Weg vorbei.

Eine Wirtschaftspolitik, die unter kapitalistischen Verhältnissen auf eine Erhöhung der Beschäftigung abzielt, muss vor allem die positiven Erwar- 
tungen der Unternehmen stabilisieren. Daher sind alle Massnahmen zu vermeiden, die zu wachsender Unsicherheit führen. Zentral ist in diesem Kontext die Stabilisierung des Preisniveaus. Dysfunktional für die Ökonomie sind Inflationen. Doch steht Europa eher vor einer Deflation als vor dem Wiederaufleben der Inflation. Deflationäre Tendenzen wirken desaströs auf die ökonomische Entwicklung: die Realverschuldung der Unternehmen steigt, die Unsicherheiten der Banken und Vermögensbesitzer erhöht sich, eine stockende Kreditvergabe wie seit Jahren in Japan ist nicht auszuschliessen und eine abwartende Haltung bei der Nachfrage nach Gütern wird durch ein sinkendes Preisniveau belohnt. Den Anker zur Stabilisierung des Preisniveaus bildet die Nominallohnentwicklung, so dass die Gewerkschaften ihrer makroökonomischen Verantwortung nur dann gerecht werden, wenn sie eine produktivitätsorientierte Lohnpolitik betreiben. Nullrunden in Deutschland wären Gift für die ökonomische Entwicklung auch der anderen europäischen Länder.

Zur "Sicherheitsproduktion“ zählt auch, dass die Unternehmen mit einer berechenbaren und allgemeinverbindlichen Lohnpolitik rechnen können. Eine Verlagerung der Lohnverhandlungen auf die Betriebsebene muss zwangsläufig zu höherer Unsicherheit und verkürzten Planungshorizonten bei den Unternehmen führen. Es gibt keinen Grund, auf die bewährten tarifpolitischen Regelungen hierzulande zugunsten der „lahmen Enten“ zu verzichten.

Die staatlichen Instanzen ihrerseits müssen eine konjunkturstabilisierende Fiskalpolitik betreiben, indem sie konjunkturbedingte Defizite hinnehmen und nicht in hektische Sparmassnahmen verfallen, die allemal zum Scheitern verurteilt sind, wenn die konjunkturelle Entwicklung nicht stabilisiert wird. Denn konjunkturelle staatliche Defizite sind vor allem ein Marktergebnis, da sie aus der Differenz zwischen Einnahmen und Ausgaben resultieren. Da sowohl die Einnahmen (z.B. Steuereinnahmen) als auch die Ausgaben (z.B. Zuschüsse zu den Sozialversicherungssystemen) stark konjunkturabhängig sind, haben Finanzminister, egal welcher politischer Couleur, auf Haushaltsdefizite nur einen begrenzten Einfluss. Sie ergeben sich, hiervon wusste der frühere Finanzminister Waigel ein Lied zu singen, als Resultat von Marktprozessen. Führen Konsolidierungsmassnahmen sogar dazu, dass bei schwachem Wachstum das weitere Wirtschaftswachstum gedämpft wird, dann wirken sie kontraproduktiv auf das angestrebte Konsolidierungsziel. Daher ist es allemal günstiger, konjunkturbedingte Defizite hinzunehmen - wie dies beispielsweise Grossbritannien und die USA in der letzten Krise mit Erfolg getan haben. Dass Lafontaine für diese „Binsenweisheit“" ins Sperrfeuer der Kritik geraten ist, zeigt, wie emotional die Diskussionen um die Staatsverschuldung (und die Inflation) hierzulande geführt werden. Das Eintreten für eine antizyklische Fiskalpolitik steht da- 
bei nicht einmal dem Ziel entgegen, die Defizite der öffentlichen Haushalte überzyklisch zu beseitigen.

Die Geldpolitik muss ebenfalls einen Beitrag zur Investitionsförderung leisten, sofern das Ziel eines stabilen Preisniveaus dadurch nicht gefährdet wird. Diese Rahmenbedingung ist zur Zeit im Euroland erfüllt, da in Europa eher Deflations- als Inflationsgefahren existieren. Die Europäische Zentralbank ist dieser Aufgabe in den letzten Monaten - im Unterschied zur Fiskalpolitik in der überwiegenden Anzahl der Teilnehmerländer - gerecht geworden. Erfreulicherweise hat sie sich von der vorübergehenden „Talfahrt" des Euro nicht irre machen lassen, sondern hat sich stärker an der Preisniveauentwicklung orientiert. Dadurch hat sie für ein investitionsfreundliches Zinsniveau gesorgt.

Letztlich kann das Beschäftigungsproblem nur über steigende Investitionen und Wachstum und/oder durch Arbeitszeitverkürzung gelöst werden. Arbeitszeitverkürzungen könnten aus den Produktivitätsfortschritten finanziert werden, um so die Lohnstückkosten stabil zu halten. Wird die Stimulierung der Investitionen ins Zentrum der Wirtschaftspolitik gerückt, dann wird auch die geläufige Unterscheidung zwischen Angebots- und Nachfragepolitik hinfällig. Was üblicherweise als Angebotspolitik zur Stärkung der Investitionstätigkeit bezeichnet ist, ist dann nämlich das entscheidende Element der Nachfragepolitik. Angebotspolitik kann neoliberal oder auch anders durchgeführt werden. Die rot-grüne Regierung hat Lafontaine aus ihren Reihen gedrängt, da man ihm (zu Recht oder nicht) das Mäntelchen des bloßen Nachfragekeynesianismus umhängt hat, der in der Tat in den siebziger Jahren gescheitert ist. ${ }^{21}$ Eine Wirtschaftspolitik, die dagegen von einem modernen Keynesianismus ausgeht, der die Trennung von Angebots- und Nachfragepolitik überwindet, wurde erst gar nicht entwickelt.

Allerdings ließe sich auch mit einer solchen Umorientierung in der Wirtschaftspolitik kein beschäftigungspolitischer Erfolg gleichsam garantieren, da die Investitionsentscheidungen stets von unternehmerischen Erwartungen abhängig sind. Auch kann die Technikwahl der Unternehmen einschließlich des Entstehens neuer Techniken nicht prognostiziert werden. Die makroökonomischen Instanzen wie die Tarifparteien, die Zentralbank und die staatlichen Instanzen können ökonomische Rahmenbedingungen schaffen, die die Sicherheit erhöhen, antizyklisch wirken und Anreize für investive Entscheidungen liefern. Ob und inwieweit die Unternehmen auf solche Rahmenbedingungen mit einer Erhöhung ihres wirtschaftlichen Engagements reagieren, ist jedoch offen. Man kann die Pferde eben nur zur Tränke führen; saufen müssen sie allein.

21 Gemeint ist hier die neoklassische Synthese, der sogenannte IS-LM-Keynesianismus. 


\section{Literatur}

Bliss, C. (1975): Capital Theory and the Distribution of Income, Amsterdam Oxford.

Bosch, G. (1998): Brauchen wir mehr Ungleichheit auf dem Arbeitsmarkt? In: WSIMitteilungen, Heft 1/1998.

DGB (Deutscher Gewerkschaftsbund) (1999): Informationen zur Wirtschafts- und Strukturpolitik. Zur Entwicklung von Löhnen, Gewinnen, Kapitalrendite und Lohnstückkosten in Deutschland. Heft 5/1999 vom 7.6.1999.

DIW-Wochenbericht (1995): Hat Westdeutschland ein Standortproblem? In: Heft 38/1995.

DIW-Wochenbericht (1997): Löhne, Wechselkurse und Wettbewerbsfähigkeit. In: Heft 30/1997.

Flassbeck, H. (1998): Reallöhne und Arbeitslosigkeit. Eine einfache empirische Widerlegung der neoklassischen Beschäftigungstheorie, in: WSI-Mitteilungen, Heft 4/1998.

Gordon, David (1995): Zwischen Kelch und Lippe: Die Rolle der Mainstream-Ökonomie bei der Gestaltung der Wirtschaftspolitik, in: PROKLA 99, S.245-270.

Hahn, F. (1982): Reflection on the Invisible Hand, in: Lloyds Bank Review, April.

Hahn, F. (1984): Die allgemeine Gleichgewichtstheorie, in: D.Bell/J. Kristol (Hrsg.), Die Krise der Wirtschaftstheorie, Berlin u.a.

Heine, M. (1989): Von der Peripherie zur Wirtschaftsmetropole - und zurück. Grundzüge einer Theorie räumlicher Agglomerationsvorteile am Beispiel Berlins, Berlin.

Heine, M./Herr, H. (1999): Volkswirtschaftslehre. Paradigmenorientierte Einführung in die Mikro- und Makroökonomie, München.

Heise, A. (1997): Neoliberale Empfehlungen zur Beschäftigungspolitik in Theorie und Praxis: Grossbritannien und Deutschland im Vergleich, in: WSI-Mitteilungen, Heft 11/1997).

International Monetary Fund (1998): Statistical Appendix, World Economic Outlook, October 1998, Washington, D.C.

iwd (Informationsdienst des Instituts der deutschen Wirtschaft) (1999): Dynamisches Defizit, in: Heft 24 vom 17.6.1999.

OECD (1996): Employment Outlook, Paris.

Samuelson, P. (1966): A Summing Up, in: The Quarterly Journal of Economics, Vol. 80.

Volz, J. (1998): Können die Niederlande ein beschäftigungspolitisches Vorbild für Deutschland sein? In: Scherrer u.a. (Hrsg.), Von den Nachbarn lernen. Wirtschafts- und Beschäftigungspolitik in Europa, Marburg. 